\title{
PSICOLOGÍA Y ASUNTOS ECONÓMICOS: UNA APROXIMACIÓN AL ESTADO DEL ARTE
}

\author{
Argemiro Alejo Riveros, Patricia Rojas Arias, \\ Universidad de Ibagüé, Ibagüé - Colombia; \\ Andrés M. Pérez-Acosta, \\ Universidad del Rosario, Bogotá - Colombia
}

\begin{abstract}
Although economic psychology has a short history it has showed an important growth in the last decades due to its importance to understand human behavior. This article addresses the state of the art in economic psychology and its tight connections with social psychology and economic science are highlighted. The paper is divided in three sections: 1 . Historical Context: origins of economic psychology, dating back to the late XIX century, and the role of pioneers such as Gabriel Tarde, Wilhelm Wundt, George Katona and Paul Albou. Additionally, this section includes a review of contributions by more recent researchers such as Fred van Raaij, Stephen E. G. Lea y Paul Webley 2. Theoretical context: the key assumptions of economic psychology are presented in this section along with some of the most influential contemporary models and the supporting empirical evidence. A clear example of the impact of this discipline is the fact that two psychologists (Herbert A. Simon and Daniel Kahneman) received the Economy Nobel Prize (1978 and 2002). 3. Metholodogical aspects: the most common used research methods and techniques in this field are reviewed. On this regard, while techniques such as open surveys and field observations are the most prominent experimental methodology in controlled environments is also used. Thanks to this methodological diversity economic psychology has succeeded to address complex themes such as money saving, unemployment, voluntary work, income tax evasion, consumer behavior and development of economic concepts in children (e.g. money).
\end{abstract}

Keywords: Behavioral economy, consumer behavior, history of psychology, theories, research methods.

\section{RESUMEN}

La psicología económica tiene una corta historia pero también un crecimiento importante en las últimas décadas por la importancia que reviste para la comprensión del comportamiento humano. En este artículo se efecta una aproximación al estado del arte de la psicología económica, resaltando su estrecha conexión tanto con la psicología social como con la ciencia económica. El artículo se divide en tres partes: 1. Contexto histórico: se examinan los orígenes de la psicología económica hacia finales del siglo XIX y el rol de pioneros como Gabriel Tarde, Wilhelm Wundt, George Katona y Paul Albou. También se examinan los aportes de investigadores más recientes como Fred van Raaij, Stephen E. G. Lea y Paul Webley 2. Contexto teórico: se presentan los supuestos claves de la psicología económica, algunos de sus modelos contemporáneos más influyentes e investigación empírica de respaldo. Una muestra clara del impacto de esta disciplina es el hecho de que dos psicólogos (Herbert A. Simon y Daniel Kahneman) recibieron el Premio Nobel de Economía (1978 y 2002). Finalmente, 3. Aspectos metodológicos: se reseñan los métodos y técnicas de investigación más frecuentemente usados en el área. Al respecto, predominan las técnicas como las encuestas abiertas y las observaciones de campo, aunque también hay presencia de la metodología experimental en ambientes controlados. Gracias a esta diversidad metodológica, la psicología económica ha podido aproximarse a temas complejos como el ahorro, el desempleo, el trabajo voluntario, la evasión de impuestos, la conducta del consumidor y el desarrollo infantil de conceptos económicos como el dinero.

Palabras Clave: economía conductual; conducta del consumidor; historia de la psicología; teorías; métodos de investigación.

Fecha recepción: 12/02/2007• Fecha aceptación: 18/05/2007

Dirección de Correspondencia:

Argemiro Alejo Riveros

Universidad de Ibagüé, Programa de Psicología

Carrera 22 Calle 67, Barrrio Ambalá, Ibagüé, Tolima - Colombia

E-mail:alejoargemiro@gmail.com
Los humanos constantemente adquiren bienes y servicios, se ven frente a situaciones ante las que se hace necesario tomar una decisión; ahorrar o comprar, endeudarse o no adquirir el producto, pagar un impuesto o evadirlo. Para la explicación de estos y otros problemas económicos se recurrió a la economía, siendo este el modelo predominante por años, con valiosas 
aportaciones pero que no eran suficientes. Todas estas decisiones son comportamientos que las personas emiten ante determinada situación, por lo tanto para su comprensión fue necesario acudir a las teorías desarrolladas por los psicólogos para la explicación del comportamiento, en este caso el económico.

En la explicación de la conducta económica se han aplicado los modelos teóricos más importantes de la psicología entre los que se encuentran la psicología del aprendizaje, las teorías cognitivas y la psicología social al igual que los métodos experimentales y descriptivos que utilizan los psicólogos en sus investigaciones. Buena parte de la investigación se ha orientado a indagar los procesos psicológicos implicados en el comportamiento económico pero igualmente se ha investigado cómo la economía afecta el comportamiento de los individuos (Lea \& Webley, 1991).

\section{CONTEXTO HISTÓRICO}

La Psicología Económica tiene una corta historia con un crecimiento importante en las últimas décadas por la importancia que reviste para la comprensión del comportamiento humano (Earl, 2005; Kahneman, 2003b). Las primeras referencias a los factores psicológicos como explicación de la conducta económica, pueden ubicarse a finales del siglo XIX con autores como Tarde (1881), aún cuando los primeros trabajos sistemáticos se iniciaron a mediados del siglo XX con George Katona ("Análisis Psicológico del Comportamiento Económico", 1965, y "Psicología de la Economía", 1979), quien realizó predicciones acerca del comportamiento de la economía en la posguerra. Posteriormente, la Psicología Económica tuvo un mayor desarrollo en Europa, donde se crearon asociaciones y se organizaron congresos (Van, 1979). En Estados Unidos, no se registraron avances en este aspecto, sólo se trabajó lo que se dio en llamar Psicología del Consumidor. Actualmente, la Psicología Económica es un área en la cual, tanto las investigaciones como el número de publicaciones y eventos a nivel internacional, han ido en aumento; su importancia es tan indiscutible, que tanto los economistas como los psicólogos y demás profesionales de las Ciencias Sociales, toman en cuenta sus hallazgos para la comprensión de los fenómenos económicos.

Quintanilla y Bonavía (2005) proponen iniciar el recuento histórico de la Psicología Económica a partir de la Escuela Marginalista o Escuela Psicológica Austriaca, con la publicación de la obra de Carl Menger "Fundamentos de la Economía Nacional" en 1871; citan como autores representativos a los austriacos Böhm-Barew y Von Weiser, el suizo Walras y el inglés Stanley Jevons, quienes "defendieron la validez universal de las leyes clásicas y el retorno al modelo liberal. Introdujeron en el análisis económico la teoría subjetiva del valor y desarrollaron el concepto de utilidad marginal por el que el valor de un bien depende de su utilidad marginal. Es decir por la satisfacción que produce la última unidad consumida de ese bien," (2005, p. 44).
Cruz (2001) plantea el debate en torno al nacimiento de la Psicología Económica como campo del conocimiento y si la sola denominación de la especialidad, es criterio para considerarla como tal. A este respecto, cita a Karl-Erik Wärneryd, quien señala la existencia de un desarrollo paralelo entre la Escuela de Austria y el trabajo de Wundt: "Los miembros de la Escuela de Austria no aguardaron el crecimiento de la ciencia de la psicología para las suposiciones psicológicas de su trabajo teórico aunque ellos comenzaron casi al mismo tiempo de cuando Wundt fundaba su laboratorio psicológico en Leipzig en 1879." (Wärneryd, 1988, citado por Cruz, 2001, p. 220).

Uno de los principales referentes históricos lo constituye el trabajo de Gabriel Tarde en 1881, completado posteriormente y convertido en el primer libro denominado "Psicología Económica"; Tarde también fue el primero en definir la especialidad; a partir de allí, se han mantenido intereses esporádicos para continuar el estudio conjunto de las dos disciplinas durante más de un siglo (Cruz, 2001; Lea, Tarpy, \& Webley, 1987; Quintanilla \& Bonavía, 2005).

Tarde (1843-1904) fue un sociólogo interesado en la conducta criminal. Sin ser psicólogo de formación, sostenía que la Sociología debía fundamentarse en la Psicología y que la realidad social era producto de estados psicológicos que se dan como resultado de la asociación de individuos. Postuló con respecto a la imitación, las tendencias del comportamiento son iniciadas por personas de estatus superior e imitadas por las de menores estatus (ley del descenso); la difusión de ideas de una población inicia lentamente para después crecer con rapidez (ley de la progresión geométrica); la cultura propia es imitada antes que lo extranjero (ley de lo propio antes que lo extraño) (Álvaro \& Garrido, 2003).

Según Denegri (2004) la teoría psicológica de la causación de conductas económicas de Tarde partía de supuestos como conducta económica, consumidor, precio, consumo, producción y capital. La conducta económica es el resultado de la acción conjugada de causas psicológicas de deseo y creencia; el consumidor es un ser hecho de deseos y apetitos que cree, con o sin razón, en la utilidad agregada del deseo cuando se consigue; el precio, lo que cuesta, lo que se desea en términos afectivos viene determinado por la intensidad del deseo. La demanda refleja la fuerza de las creencias que son los mitos relacionados con una época determinada; el consumo fluctúa constantemente según las formas en que esos mitos adquieren poder, ambición y desafíos, lo que se amplía por la imitación; la producción depende de la invención y del trabajo, los cuales a su vez vienen determinados por las necesidades y el capital es el resultado de la suma de beneficios (manifestados según las creencias del momento) que generan los deseos.

Ya en el siglo XX, George Katona logra un reconocimiento de la Psicología Económica como disciplina autónoma tanto conceptual como metodológicamente. Su tesis central postula que los procesos económicos surgen directamente del comportamiento humano, como resultado del comportamiento de las personas y están influenciados por 
diferentes reglas de conducta. Por esto, es necesario estudiar los motivos, actitudes y expectativas, tanto de los consumidores como de los empresarios, frente a las conductas de gasto, ahorro e inversión. Para este autor es necesaria la utilización de principios psicológicos y métodos de investigación tanto económicos como humanos y sociales, además de la preparación psicológica de la política económica (Katona, 1979, 1974).

Katona propende por una economía que estudie los procesos tal como se presentan bajo condiciones especiales, por los descubrimientos empíricos basados en la observación del comportamiento, sin asumir la existencia de una conducta racional, ni ideales o dogmas. Define su campo de interés en los procesos económicos de Estados Unidos durante el siglo $\mathrm{XX}$, porque es la información que tiene disponible; al respecto manifiesta que "la investigación empírica es lenta y modesta". En sus estudios, parte de la consideración de los procesos económicos como fenómenos influidos por decisiones humanas y del interés por investigar las condiciones y limites de dichas decisiones. Otro aspecto considerado por este autor es la necesidad de complementar los datos macroeconómicos con los datos microeconómicos, ya que los datos macroeconómicos "no nos dicen toda la verdad" (Katona, 1965, 1974).

Para Quintanilla y Bonavía (2005), las principales contribuciones de Katona fueron: 1. Consolidar un equipo de investigadores, desarrollar numerosas investigaciones desde el laboratorio que él dirigió en Ann Arbor, y pretender realizar contribuciones a la explicación del comportamiento económico desde la Psicología, sin pretender una explicación exclusivamente psicológica de la Economía. 2. Defender la sociedad de consumo como resultado del trabajo y ahorro de las clases medias, y no como la dominación de una clase poco numerosa de privilegiados.

Dentro del desarrollo histórico de la Psicología Económica también se cita frecuentemente al francés Paul Albou (1984) y su "modelo ternario y gráfico provisional", el cual puede descomponerse en dos partes: bases contextuales y bases psicológicas. Las bases contextuales corresponden al contexto económico, político y sociocultural de una sociedad particular en una época determinada. Las bases psicológicas, a su vez, se encuentran compuestas por tres sectores de similar importancia: el conativo (actividades económicas); el afectivo (sentimientos) vinculado con el conativo por medio de la motivación; y el cognitivo, que permite la percepción e interpretación de la realidad (Denegri, 2004, Quintanilla \& Bonavía, 2005).

Por su parte, Fred van Raaij (1990) propone el modelo de integración, donde se insiste en tener en cuenta la retroalimentación entre la conducta económica y las condiciones del medio. Incluye elementos como el bienestar subjetivo, el cual puede comprender la satisfacción o descontento asociado a la compra o sus reclamaciones, que es la consecuencia directa de la conducta económica. La conducta económica es la decisión humana de elección entre alternativas de uso de recursos tales como dinero, tiempo, esfuerzo, espacio, recursos material y energía para satisfacer necesidades.

Uno de los desarrollos teóricos de la Psicología Económica más recientes se ha llevado a cabo en la Universidad de Exeter (Inglaterra), donde los investigadores Lea et al. (1987) han propuesto el paradigma de la causación dual, considerando que la conducta económica esta sometida a ella, es decir, algunas conductas económicas determinan el curso de los asuntos económicos (Burgoyne, Routh, \& Ellis 1999), lo mismo que la economía, como realidad social, influye sobre la conducta humana (Palomar \& Pérez, 2003; Moyano \& Barría, 2006; Gaines \& Cho, 2004). Destacan estos autores la interdependencia entre la economía y el comportamiento de los individuos y enfatizan en la necesidad de no centrarse en el debate tradicional de si la conducta económica es o no racional, sino mas bien, propender por el acceso de los individuos a la mayor cantidad de información posible para que conozcan y comprendan los efectos que su conducta tiene en la economía y cómo son influidos por esta (Denegri, 2004; Quintanilla \& Bonavía, 2005).

Por último, es importante advertir que psicólogos como Herbert Simon (en 1978) y Daniel Kahneman (en 2002) han obtenido Premios Nobel de Economía (Ardila, 2001; Cruz, 2003; Kahneman, 2003b), básicamente por la investigación sobre el papel de la cognición en las decisiones y la racionalidad limitada (Kahneman, 2003a; Lovallo \& Kahneman, 2000). Los economistas recurren cada vez más a las investigaciones de la Psicología Económica y de la Psicología General para la comprensión y predicción de fenómenos económicos. Este es un largo camino que apenas se ha empezado a recorrer.

\section{CONTEXTO TEÓRICO}

El estudio del comportamiento económico es complejo ya que no hay una teoría económica que permita explicar los fenómenos globales del desarrollo, ni las crecientes disparidades sociales porque ignora la estructura social y sus mutaciones y las cambiantes relaciones de poder que emergen de todo ello (Bunge, 1985).

La idea de la Psicología Económica como un esfuerzo interdisciplinario se basa en la pretensión común, tanto de la Economía como de la Psicología, de estudiar la conducta humana. La Psicología Económica es el cuerpo de conocimientos resultado de la investigación interdisciplinaria; sus investigadores reconocen que el problema es económico y psicológico y que se deben emplear ambos métodos para estudiarlo (Lea et al., 1987). De esta manera, se deben garantizar elementos de formación comunes básicos de Psicología y de Economía para abordar temáticas específicas de investigación en el campo.

El ser humano en la sociedad occidental se desenvuelve exclusivamente dentro de contextos regulados 
económicamente, hasta el punto de que toda su actividad corresponde a modelos y patrones de interacción entre agentes de orden económico de amplia aceptación y de incuestionable existencia. Retomando las palabras de Katona (1965), diríamos que: "los procesos económicos surgen directamente del comportamiento humano", esto es, son inherentes a la condición de conformarse como seres determinados socialmente. Katona parte de algunos postulados básicos como que la psicología es una disciplina empírica donde el comportamiento es una entidad susceptible de medida y análisis a nivel molar y molecular, que se caracteriza por su plasticidad dentro de unos extensos límites y en su análisis hace uso de las variables participantes.

La conducta económica se manifiesta de muchas formas, tanto que sería casi imposible describir todos los tópicos de investigación de la Psicología Económica. Fred van Raaij (1990) propone un total de once tópicos:

1. El comportamiento del consumidor ha sido muy desarrollado, especialmente como aplicación al marketing.

2. La investigación en Publicidad, principalmente como parte del marketing, esta relacionada con el impacto de la publicidad en actitudes, cogniciones y conductas. La interacción entre factores afectivos y cognitivos (ver Pérez-Acosta, 2004, 2006; Rial, Varela \& García, 2001; Baker, 1999).

3. El estudio de la conducta empresarial tiene similitudes con la conducta del consumidor, especialmente en las conductas de compra e inversión de empresarios. Se incluyen investigaciones sobre decisiones organizacionales con conceptos como satisfacción y nivel de aspiraciones.

4. Las conductas de inversión, la percepción del riesgo y la toma de ellos, originan especial atención. Los inversionistas frecuentemente toman riesgos bajo condiciones de incertidumbre. Los rumores y recomendaciones de expertos podrían tener características completamente diferentes.

5. Los impuestos y las conductas de evasión son un campo de investigación interesante en los años recientes (King \& Sheffrin, 2002).

6. La producción familiar como tópico económico olvidado. Las estadísticas oficiales no cuentan el trabajo casero, una importante área de producción, especialmente, durante las recesiones. La producción casera esta considerada como economía informal.

7. La energía y el medio ambiente fueron tópicos populares en la década de los años 70 para investigar qué clase de personas tenían en cuenta sus efectos en el consumo y en la producción. La psicología económica podría evaluar, por ejemplo, las actitudes hacia el consumo y las respuestas a los programas de conservación.
8. Katona (1979) investigó el efecto del sentimiento del consumidor (evaluaciones y expectativas) en relación con el gasto, el ahorro y la solicitud de préstamos. En períodos de recesión con bajos ingresos, las personas tendían a ahorrar más y a gastar menos. Esto es contrario a las hipótesis de Keynes. La explicación es que en los períodos de recesión la gente tiene incertidumbre sobre el futuro y desea crear un amortiguador financiero.

9. El desempleo es el mayor problema de muchas sociedades. La psicología económica investiga la experiencia de desempleo, la búsqueda de trabajo y la conducta de gasto del desempleado. Un aspecto especial del Journal of Economic Psychology ha sido el desarrollo de estudios sobre desempleo.

10. La percepción de la inflación y sus efectos en el gasto y el ahorro, incluyendo ilusiones monetarias, son tópicos de investigación de la Psicología Económica (Kahneman, Ritov, \& Schkade, 1999; Cruz, 1998).

11. La satisfacción del consumidor después de la compra es un concepto relativo, centrado en la discrepancia entre las expectativas y la ejecución actual de un producto o servicio.

Denegri (2004) postula otros once elementos como necesarios para la comprensión de la compleja conducta económica:

1. El comportamiento no tiene lugar en el vacío o separado de otros aspectos del comportamiento humano.

2. Las decisiones económicas implican dinero, tiempo y esfuerzo para obtener bienes y servicios e involucran resolver la tensión entre ahorro y/o gasto. Cualquier conducta que involucre elecciones o transacciones entre gasto o inversión y que considere futuras ganancias y beneficios, es una decisión económica.

3. Las decisiones económicas se caracterizan por las renuncias que debe hacer el individuo (costo de oportunidad), la evaluación de los beneficios presentes o futuros de un desembolso (gasto o ahorro), la evaluación de los beneficios esperados de algunas alternativas (costo/beneficio) y la realización de la conducta concreta.

4. Los criterios usuales para una decisión económica son el financiero, el temporal, la evaluación del esfuerzo requerido para conseguir las expectativas presentes o futuras de bienestar.

5. Los determinantes de las decisiones económicas incluyen factores personales, sociales, culturales, situacionales y también factores económicos generales, que estimulan o inhiben la conducta (Chien \& DeVaney, 2001).

6. Los factores personales son: características de personalidad del individuo, estilo de vida personal y familiar, normas y 
valores de su cultura, niveles de comprensión del mundo económico. Estos factores son influidos, a su vez, por la región de residencia, subcultura, grupo etáreo y sexo (Roberts \& Jones, 2001).

7. Los factores sociales y culturales incluyen el estrato socioeconómico de pertenencia, las expectativas sociales y las características globales del sistema político y cultural en el que esta inserto el individuo.

8. Los factores situacionales son las condiciones y circunstancias que normalmente limitan las decisiones económicas: ingreso disponible, tamaño de la familia, tipo de hogar, situaciones de mercado, existencia de ofertas.

9. Los factores económicos generales se relacionan con la percepción del estado económico: tasas de inflación e interés, tasa de cesantía, equidad de la distribución del ingreso y políticas económicas. Estos factores generales provocan actitudes pesimistas u optimistas y generan expectativas que influyen en las decisiones de gastar, ahorrar o invertir.

10. Las consecuencias de las decisiones económicas son la satisfacción, el bienestar o el descontento de los individuos.

11. Las decisiones económicas sirven de experiencias de aprendizaje que pueden influir en las próximas decisiones. Estas experiencias pueden ser directas o vicarias.

B. F. Skinner (1977), en su clásica obra "Ciencia y Conducta Humana", destaca que la teoría económica tradicional se ha inclinado a utilizar el principio de máximos y mínimos, al deducir la conducta del individuo que efectúa transacciones económicas a partir de los datos que se desprenden del grupo. Este procedimiento condujo al Homo economicus, concepto propio de la teoría económica del siglo XIX, que se fundaba justamente en la conducta necesaria para explicar el conjunto de los hechos del gran grupo. Esta ficción explicativa ya no desempeña ningún papel importante en la teoría económica. Skinner también plantea desde la Psicología una relación entre el refuerzo operante y la retribución económica, en los siguientes términos: "Como simple ejemplo del control económico, se induce a un individuo a llevar a cabo un trabajo reforzándolo con dinero o bienes. El que controla hace que el pago de la retribución sea contingente con respecto a la realización del trabajo. Sin embargo, en la realidad el proceso no suele ser tan simple. Cuando damos a alguien una propina o le pagamos por realizar un pequeño servicio y, por tanto, aumentamos la probabilidad de que lo realice en el futuro, no obramos de modo muy distinto a como lo hacemos en un laboratorio al estudiar el refuerzo operante" (p. 406).

Pereira, Angel y de la Espriella (1991), señalan la relevancia que tuvo la aplicación de los principios de refuerzo en ambientes institucionales a través de los programas de economía de fichas en contextos clínicos y educativos, lo que permitió un análisis económico de tipo práctico que potenció el desarrollo de conceptos centrales en la investigación básica tales como la
Ley de Igualación. A su vez, la Ley de Igualación se emplea para analizar la conducta de elección y los procesos de toma de decisión (Clavijo, 1997). Los procesos de decisión se pueden trabajar desde dos perspectivas: desde la teoría conductual o desde la teoría cognitiva más específicamente desde el procesamiento de información (Clavijo, 1997; Quintanilla \& Bonavía, 2005).

La Psicología Social es el estudio científico de cómo piensan las personas, como se influyen y cómo se relacionan unas con otras. Resalta la influencia del medio social en el comportamiento, busca entender, definir y determinar patrones de conducta que permitan una delimitación de los factores que puede afectar nuestras actuaciones cotidianas. De esta manera, la esencia de la Psicología reposa en la búsqueda de modelos que faciliten la generalización de las variables que explican las conductas (Worchel, Cooper, Goethals, \& Olson, 2002). Los conceptos trabajados en las investigaciones de Psicología Económica incluyen las temáticas de actitudes, atribuciones, sesgos explicativos (Kahneman, 2003a) y procesos relacionados con la memoria, entre otros, trabajadas por la Psicología Social. La diversidad de comportamientos humanos presentes en la gran variedad de situaciones de interacción social permite a la Psicología Social explicar el comportamiento humano en las transacciones económicas.

En cuanto a formación de actitudes encontramos teorías explicativas como la del condicionamiento clásico de preferencias y emocional y su modificación a partir de estos o del aprendizaje operante (Cruz \& Pérez-Acosta, 2002; Hammer, 1997; Lea et al., 1987), que permiten comprender los comportamientos económicos en general y han sido utilizadas, especialmente, para evaluar los efectos de la publicidad, las actitudes tambien se han relacionado con el endeudamiento (Chien \& DeVaney, 2001). El nivel de publicidad al que los consumidores se exponen varía según el tiempo y el lugar. Se afirma que la presencia en todas partes de la publicidad puede inducir cambios psicológicos generales, especialmente en los niños. La publicidad puede hacer a la gente más materialista $\mathrm{o}$ más preocupada por una creciente cantidad de productos. (Lea et al., 1987).

La Psicología Económica "se preocupa del estudio del comportamiento económico, de las variables que inciden en la toma de decisiones económicas individuales y colectivas y las formas como las personas comprenden el mundo de la economía y sus variaciones" (Denegri, 2004). Desde esta perspectiva, se han planteado una gran variedad de estudios para determinar si los factores comprometidos son, efectivamente, causantes o generadores de determinadas conductas. Al respecto se ha concluido que existe una relación bidireccional entre los efectos de la economía y el comportamiento individual económico, el cual comprende las acciones, deseos y pensamientos a la hora de decidir entre gasto o ahorro (Denegri, 2004; Katona, 1979; Lea et al., 1987; van Raaij, 1990).

Desde los años sesenta, las características psicológicas que determinan los elementos constitutivos del comportamiento 
económico, en particular la conducta de compra, han venido cobrando importancia dentro de los estudios realizados por la Psicología Económica en los Estados Unidos, intentando con esto detallar y predecir lo que los consumidores desean en el momento de obtener bienes de consumo.

La importancia de dichas investigaciones ha sido proveer de diversos esquemas, modelos y patrones que, en la práctica, permiten a los economistas realizar proyecciones y supuestos acerca del comportamiento macroeconómico. Es decir, modelos que sirvan como herramienta de aplicación útil a la hora de crear estrategias de desarrollo económico y de contingencia en empresas y países.

La realización de estos estudios beneficia no sólo a los psicólogos en su afán por comprender el comportamiento humano, sino también a los economistas e individuos particulares que tendrían la posibilidad de contar con modelos que permiten efectuar predicciones a más largo plazo. Actualmente, los teóricos de la economía utilizan modelos psicológicos para explicar los procesos económicos y prever los cambios en el entorno (Elster, 2003).

El tema del comportamiento del consumidor se refiere a todos aquellos procesos que intervienen cuando una persona o grupo selecciona, adquiere, usa o desecha productos, servicios, ideas o experiencias para satisfacer sus necesidades y deseos. El comportamiento del consumidor desde un punto de vista científico, es el conjunto de actividades o reacciones de una persona ante los estímulos; además de ser un término descriptivo para referirse a las acciones de una persona ante estímulos derivados de actividades comerciales que se traducen en la adquisición y el uso de bienes y servicios, incluyendo los procesos de decisión que preceden y determinan aquellos actos.

El estudio del comportamiento del consumidor nos hace conocedores de las sutiles influencias que persuaden para hacer las elecciones de productos o servicios. Para entender el comportamiento humano en general, resulta importante entender las influencias internas y externas que llevan a los individuos a actuar de determinada manera frente al consumo.

El tema del comportamiento del consumidor incluye la actitud que tienen los consumidores frente a las marcas del mercado y de qué forma influyen éstas, dentro del comportamiento de consumo. Sobre este tópico, se hace un número considerable de investigaciones, cuyos temas generales son: La lealtad hacia la marca, tema principal de cuatro de las investigaciones revisadas, vista esta como el compromiso que tiene un cliente para adquirir nuevamente un servicio o un producto de su predilección, pese a las influencias situacionales y los intentos del marketing por generar cambios en el comportamiento (Fuentes, Luque, Montoso, \& Cañadas, 2004; Velandia, 2002). La influencia de la marca sobre el comportamiento del consumidor es motivo de investigación de otros dos estudios, y el resto de las investigaciones se ocupan de otras temáticas, tales como la implicación con la marca, definida como el valor percibido de un objeto concreto que se manifiesta como un interés por dicho objeto (Varela, Rial, García, Briana, \& Olea, 1996); las extensiones de marca y la formación de actitudes hacia la marca. Dentro de esta última, se hace referencia al posicionamiento, explicándose claramente cómo éste, representa una parte considerable de la identidad de una marca, en la medida en que permite diferenciarla de las marcas competidoras (Hartmann, Apaolaza, \& Forcada, 2004).

En cuanto al tema de compra impulsiva, encontramos que existen diferentes definiciones del término consumo, las cuales han sido formuladas por distintos autores a lo largo de la historia (Boddington \& Kemp, 1999). Según lo citado por Ortega y Rodríguez-Vargas (2003), una de las más amplias es la que incorpora a la teoría del comportamiento del consumidor elementos ecológico-ambientales asociados a las actividades de obtención y uso. En el otro extremo está la que restringe el consumo al término compra.

En cualquier caso, buena parte de los estudios conciben el consumo en términos de sus relaciones de compra, la adquisición, el gasto económico, la obtención, el uso y la posesión de bienes y servicios. En economía, el consumo es visto como resultado de la maximización de la función de utilidad, sujeta a ciertas restricciones de carácter presupuestario, donde el concepto de utilidad en sí mismo, no constituye el foco de interés. También, utilidad y actitud presentan similitudes conceptuales, ambos términos aluden a la capacidad de satisfacción de necesidades que tienen los bienes y servicios, al tiempo que reflejan preferencias e influyen en el comportamiento. Luna (1998), citado por Ortega y Rodríguez-Vargas (2004), plantea que desde la Psicología Económica se ha optado por el término maximización, refiriéndose a la conducta que busca optimizar un resultado en lugar de analizar su racionalidad o irracionalidad.

Mientras que desde la teoría económica "el comportamiento racional implica que los consumidores eligen alternativas de consumo que proporcionan los mayores beneficios, dado su presupuesto, los modelos psicológicos no suponen una función de utilidad constante, sino que toman en cuenta las diferencias individuales para definir una función de utilidad subjetiva y, de esta forma, puede brindar una explicación más realista del comportamiento" (Ortega y Rodríguez-Vargas, 2004, p. 123).

Bajo la perspectiva psicológica, una de las aproximaciones al estudio de los hábitos de compra ha distinguido una concepción bipolar entre reflexividad e impulsividad del comportamiento de consumo. Denegri, Palavecinos, Ripio y Yáñez, (1999), citados por Ortega y Rodríguez-Vargas (2004), parten de esta concepción bipolar, distinguiendo que la definición de la conducta de compra reflexiva toma como base en su definición el consumo racional y planificado, que abarca actos dirigidos a una gestión eficiente de los recursos económicos disponibles, lo que implica jerarquización de las necesidades de compra, ordenar los productos según su importancia, previsión de gastos, entre otros; por el contrario, de acuerdo con Luna (1998), citado por Ortega y Rodríguez- 
Vargas (2004), la conducta de compra impulsiva vendría siendo caracterizada por la ausencia de planificación.

Sumado a esto, los investigadores Chien-Huang y Hung-Ming (2005) manifiestan que la compra impulsiva se define como "una compra imprevista" que se ha caracterizado por la toma de decisiones relativamente rápida, hacia la posesión inmediata. En cualquier caso, Luna-Arocas (1998) y Denegri (1999), citados por Ortega y Rodríguez-Vargas (2003), manifiestan que todas las definiciones de compra impulsiva han contemplado la ausencia de previsión en el acto de consumo y la escasa atención a los recursos financieros disponibles.

En los resultados de la compra compulsiva pueden incluirse el endeudamiento y los consiguientes problemas legales, el estrés y los conflictos de pareja (Ortega \& Rodríguez-Vargas, 2004). Los estudios realizados sobre la compra por impulso mantienen la tendencia a incorporar en sus indicadores, variables de deuda y gasto (Roberts \& Jones, 2001).

En esta misma línea, Chien-Huang y Hung-Ming (2005) plantean que, en los adolescentes, el comportamiento impulsivo se ha visto generalmente como ineficaz y que las diferencias individuales en el impulso se encuentran relacionadas con un número de comportamientos sociales relevantes, incluyendo la agresión.

Por otro lado, en los estudios sobre los procesos o conductas de compra se ha utilizado uno de los modelos más divulgados y aceptado sobre la conducta económica, propuesto por van Raaij (1990). Este Modelo integrador de la conducta económica, retoma en su formulación la necesidad manifestada por Katona de considerar los círculos de retroalimentación entre la conducta económica y las condiciones del medio. El modelo se sintetiza en los siguientes elementos y relaciones: a) factor personal (los valores, las aspiraciones, los estilos cognitivos y actitudes, las variables sociodemográficas); b) situación, ambiente percibido (clima en los negocios, expectativas de los consumidores sobre oscilaciones en los precios o sobre el reparto de los ingresos, ideas sobre la posición social de los grupos de referencia); c) conducta económica (conductas de consumo sea activo o pasivo. Ejemplo: ahorrar); d) medio económico (finanzas personales, fuentes de ingreso, relacionadas con el empleo, la situación del mercado, nivel de recesión o de expansión económica); e) bienestar subjetivo (satisfacción o el descontento asociado a una compra); f) contexto general (situación financiera en la que se desenvuelven los individuos); g) descontento social (nivel de satisfacción de los individuos en relación con sus conductas económicas).

Por otra parte, es importante resaltar el papel de la motivación que en este caso, es un comportamiento de la persona para conseguir ya sea un bien o un servicio. Los teóricos distinguen varias clases de motivos: biológicos (fisiológicos): sed, hambre, sexo; psicológicos: obtención social, status; aprendidos: normas y deseos e instintivos. Respecto a la motivación y a las necesidades del consumidor es indispensable encontrar una definición de necesidad la cual puede formularse como la diferencia o discrepancia entre el estado real y el deseado.

Otro factor que es importante en el proceso de compra es el nivel o estrato socioeconómico, tomado en cuenta por las investigaciones, porque es el resultado del desarrollo económico que marca una pauta importante en el desenvolvimiento del comportamiento de compra.

La mayor parte de las problemáticas encontradas en las investigaciones relacionadas con compra impulsiva abarcan los factores que influyen en la compra, la tendencia a la compra impulsiva y la percepción hacia las decisiones de compra; por ejemplo: "¿Influyen las características personales en la tendencia de compra impulsiva en los adolescentes de Taiwán?" (Chien-Huang \& Hung-Ming, 2005) y "¿Qué factores del consumidor influyen en la percepción subjetiva de su situación financiera?" (Vidal, 1996).

Otros estudios buscan establecer los conocimientos y las percepciones de los consumidores ante determinado producto (Freire, Rodríguez, Rodríguez, \& Sánchez, 2003), así como evaluar el estado de endeudamiento, la percepción subjetiva de la situación financiera, los hábitos y las conductas de consumo y el bienestar subjetivo asociado a estos (Ortega \& Rodríguez-Vargas, 2004).

\section{ASPECTOS METODOLÓGICOS}

Si bien como disciplinas, tanto la Economía como la Psicología tienen el mismo origen en los filósofos empiristas, la Economía parte de la formulación de una teoría y de ahí, mediante un proceso de deducción, explica los procesos y comportamientos económicos; en tanto que, la Psicología parte de los datos empíricos para ir construyendo las teorías (ver Cruz, 2001).

La Economía tiene una historia muy corta en la tradición experimental mientras que la historia de la Psicología desde sus inicios está marcada por ella. Lo anterior, no quiere decir que los economistas no se basen en datos para hacer sus predicciones, sino que difieren en el énfasis que se da al uso de la experimentación en ambas disciplinas (Lea \& Webley, 1991). Esto explica el hecho de habérseles concedido premios Nobel a algunos economistas que han desarrollado estrategias experimentales aplicadas a la Economía. Aún así, esto es extraño para la mayoría de los economistas y no es una idea generalizada entre los especialistas.

La gran variedad de comportamientos económicos clasificables como posibles problemas de estudio y las estrategias de investigación que aportan la Psicología y la Economía conforman un amplio rango de métodos para indagar acerca del comportamiento económico tanto de individuos tomados como unidad de análisis, como del resultado de la actividad económica de todo un grupo social. En primer lugar, las investigaciones se podrían identificar por 
el nivel de análisis que pretenden hacer, esto es, a nivel micro o macroeconómico. En segunda instancia, se considera el nivel de intervención que se propone el investigador: el análisis conceptual, la observación directa del comportamiento, el reporte verbal, entre otros. Un tercer parámetro relacionado con los anteriores, lo constituye la orientación que se le da a la investigación, ya sea de tipo básico o aplicado (Cruz \& PérezAcosta, 2003; van Raaij, 1990).

A nivel de investigación aplicada, las encuestas se constituyen en los instrumentos más utilizados en la investigación en Psicología Económica. Se emplean cuestionarios para recolectar información, dada su buena relación de costobeneficio. En algunas investigaciones se opta por la entrevista personal, convocada o personal domiciliaria, debido a la necesidad de utilizar material de apoyo; por ejemplo tarjetas con el nombre y el logotipo de cada una de las marcas incluidas en el estudio (ver Lea et al., 1987).

También se utilizan encuestas electrónicas, en donde se incluyen criterios para la medición de las actitudes de los participantes, distintos tipos de baterías de ítems y escalas. La estructuración de las encuestas se realiza de acuerdo con formatos de escala tipo Likert y Diferencial Semántico, para ordenación en función de las preferencias; o de tipo Denominaciones de Precios comprendidas entre cantidades asignadas. No obstante, priman los instrumentos que incluyen dentro de sus adaptaciones o diseños las escalas con formato tipo Likert de diferentes puntos, las cuales son una forma de presentar las posibles respuestas a una pregunta que conduce a una variable ordinal (Grau, Correa, \& Rojas, 1999).

Específicamente, en las investigaciones realizadas sobre compra impulsiva, han sido relacionadas variables como: El ingreso económico del grupo familiar, definido de acuerdo con las particularidades del contexto en diferentes niveles, generalmente tres: alto, medio y bajo; el sexo en dos niveles: masculino y femenino; la preocupación por las prestaciones adicionales que configuran el producto, la previsión y planificación del proceso de compra, la consideración del precio del producto, los indicadores de conductas impulsivas de consumo, el papel activador del pago, el estado de endeudamiento, la percepción subjetiva de la situación financiera, los hábitos y conductas de consumo, el bienestar subjetivo, la actitud hacia el endeudamiento y las variables sociodemográficas relevantes (Chien-Huang \& Hung-Ming, 2005).

La psicología económica es un área de confluencia de la psicología y la economía que tiene sus inicios en Europa y durante parte del siglo XX no trascendió a otras regiones, actualmente presenta un crecimiento importante si se tiene en cuenta el número de publicaciones y participación de profesionales de las ciencias sociales en la explicación de los temas económicos. Las diferencias de métodos y formas de abordar la conducta económica en psicología y en economía han permitido combinar los métodos de aproximación que provienen de ambas disciplinas; la psicología más orientada a la investigación experimental y la economía más a la elaboración de teorías. No obstante es necesario seguir trabajando en el desarrollo metodológìco y teórico que permitan una mejor explicación de la conducta económica tanto en el área básica como aplicada.

La investigación en conducta económica en temas como el endeudamiento, la inversión, el ahorro, la evasión de impuestos y la compra compulsiva requieren mayor desarrollo; igualmente en la explicación de las preferencias del consumidor es necesario implementar métodos de investigación que permitan generar estrategias de aplicación del aprendizaje asociativo a la publicidad (Froufe \& Sierra, 1998). En general se observa un desarrollo importante en la aplicación de los modelos teóricos psicológicos a la explicación de la conducta económica siendo necesario ampliar la investigación que permita la aplicación a situaciones reales.

\section{REFERENCIAS}

Albou, P. (1984). Lapsychologie économique. Paris, Francia: Presses Universitaries de France.

Alvaro, J. L., \& Garrido, A. (2003). Psicología social. Perspectivas psicológicas y sociológicas. Madrid: McGraw Hill.

Ardila, R. (2001). Herbert A. Simon (1916-2001) Psicólogo Premio Nobel. Revista Latinoamericana de Psicología, 33, 223224.

Baker, W. E. (1999). When can affective conditioning and mere exposure directly influence brand choice. Journal of Advertising, 28, 31- 46.

Boddington, L., \& Kemp, S. (1999). Student debt, attitudes towards debt, impulsive buying, and financial management. New Zealand Journal of Psychology, 28, 89-93.

Bunge, M. (1985). Economíay filosofía. Madrid: Tecnos.

Burgoyne, C. B., Routh, D. A., \& Ellis, A. M. (1999). The transition to the Euro: Some perspectives from economic psychology. Journal of Consumer Policy, 22, 91-116.

Chien-Huang, L., \& Hung-Ming, L. (2005). The effect of individual differences on adolescents' impulsive buying behavior. Adolescence, 40, 215-223.

Chien, Y., \& DeVaney, S. A. (2001). The effects of credit attitude and socioeconomic factors on credit card and installment debt. The Journal of Consumer Affairs, 35, 179162.

Clavijo, A. (1997). El estudio de la elección en condiciones de incertidumbre. Suma Psicológica, 4, 57-77.

Cruz, J. E. (1998). La moneda y su representación gráfica en el recuerdo libre. El caso de menores Colombianos. Suma Psicológica, 5, 21-46.

Cruz, J. E. (2001). Psicología económica. Suma Psicológica, 8, 213-236.

Cruz, J. E. (2003). Daniel Kahneman : un nuevo premio Nobel de economía para la psicología. Revista Latinoamericana de Psicologia, 35, 117-121.

Cruz, J. E., \& Pérez-Acosta, A. M. (2002). Técnicas experimentales aplicadas al condicionamiento clásico de preferencias en el comportamiento del consumidor. Acta Colombiana de Psicología, 8, 75-87. 
Cruz, J. E., \& Pérez-Acosta, A. M. (2003). Conceptos de Condicionamiento Clásico en los Campos Básico y Aplicado. Interdisciplinaria, 20, 205-227.

Denegri, M. (2004). Introducción a la psicología económica. Temuco, Chile: Universidad de la Frontera (documento no publicado).

Earl, P. E. (2005). Economics and psychology in the twentyfirst century. Cambridge Journal of Economics, 29, 909-926.

Elster, J. (2003). Teoría de la elección racional y sus rivales. En J. Elster, R. Inglehart, \& R. Eisler, (Eds.), Reflexiones sobre la investigación en ciencias sociales y estudios políticos. Bogotá: Universidad Nacional de Colombia.

Freire, V., Rodríguez, J. J., Rodríguez Z. M., \& Sánchez, J. C. (2003). El mercado de un queso ecológico (Río Cuarto, Argentina) actitud de los consumidores. Archivos de Zootecnia, 52(199), 408-415.

Froufe, M., \& Sierra, B. (1998). Condicionamiento clásico de preferencias: implicaciones para la publicidad. Revista de Psicología Generaly Aplicada, 51, 85-104.

Fuentes, F., Luque, T., Montoro, F., Cañadas, P. (2004). Efectos del valor de la marca sobre la lealtad y el precio atribuido por el consumidor. Libro de Ponencias del XVI Encuentro de Profesores Universitarios de Marketing, Alicante (España).

Gaines B. J., \& Cho, W. K. T. (2004). On California's 1920 Alien Land Law: The Psychology and Economics of Racial Discrimination. State Politics \& Policy Quarterly, 4, 271-293.

Grau, R., Correa, C., \& Rojas, M. (1999). Metodología de la investigación. Ibagué, Colombia: Corporación Universitaria de Ibagué.

Hammer, M. (1997). Effects of US-alone presentations on human evaluative conditioning. Learning and Motivation, 28, 491-509.

Hartmann, P., Apaolaza, V., \& Forcada, J. (2004) La influencia del posicionamiento verde en la actitud hacia la marca. Libro de Ponencias XVI Encuentro de Profesores Universitarios de Marketing, Alicante (España).

Kahneman D. (2003a). Mapas de racionalidad limitada: psicología para una economía conductual. Revista Asturiana de Economía, 28, 181-225.

Kahneman D. (2003b). A psychological perspective on economics. The American Economic Review, 93, 162-168.

Kahneman D., Ritov, I., \& Schkade, D. (1999) Economic preferences or attitude expressions?: An analysis of dollar responses to public issues. Journal of Risk and Uncertainty, 19, 203-235.

Katona, G. (1965). Análisis psicológico del comportamiento económico. Madrid: Editorial Rialp.

Katona, G. (1974). Psychology and Consumer Economics. Journal of Consumer Research, 1, 1-8.

Katona, G. (1979). Psicología de la economía. Buenos Aires: Editorial El Ateneo.

King, S., \& Sheffrin, S. M. (2002). Tax Evasion and Equity Theory: An Investigative Approach. International Tax and Public Finance, 9, 505-521.

Lea, S., Tarpy, R., \& Webley, P. (1987). The individual in the economy. A textbook of economic psychology. New York: Cambrigde University Press.
Lea, S., \& Webley, P. (1991). Psicología económica: una introducción a un nuevo campo interdisciplinario. Informe interno de la Universidad de Exeter, Inglaterra (traducción: Andrés M. Pérez-Acosta).

Lovallo, D., \& Kahneman D. (2000). Living with uncertainty: attractiveness and resolution timing. Journal of Behavioral Decision Making, 13, 179-190.

Moyano, E., \& Barría, R. (2006). Suicidio y producto interno bruto (PIB) en Chile: hacia un modelo predictivo. Revista Latinoamericana de Psicología, 38, 343-359.

Ortega, V., \& Rodríguez-Vargas, J.C. (2003). Escala de hábitos y conductas de consumo: evidencias sobre dimensionalidad. Revista Internacional de Psicología Clínica y de la Salud / International Journal of Clinical and Health Psychology, 4(1), 121-136.

Ortega, V., \& Rodríguez-Vargas, J.C. (2004). Consumo y deuda en adultos jóvenes: evaluación desde un modelo integrador de la conducta económica. Revista Latinoamericana de Psicología, 37(1), $95-118$.

Palomar, J., \& Pérez, A. (2003). Un solo rostro y tres maneras de mirarlo: el significado de "pobreza" según el nivel socioeconómico. Revista Latinoamericana de Psicología, 35, 27 39.

Pereira, C., Angel, E., \& de la Espriella, C. (1991). Teorías conductuales de la motivación. En M. V. Mankeliunas (Ed.), Psicología de la motivación (pp. 384-419). México: Editorial Trillas.

Pérez-Acosta, A. M. (2004). Prueba experimental del efecto de preferencia de marcas pioneras. Suma Psicológica, 11, 205220.

Pérez-Acosta, A. M. (2006). Prueba empírica de un modelo asociativo de recuerdo de marcas publicitarias con datos de mercado real. Suma Psicológica, 13, 85-95.

Rial, A., Varela, J., \& García, A. (2001). Identificación de las variables que determinan la evaluación de una extensión de marca. Psicothema, 13, 284-289.

Roberts, J., \& Jones, E. (2001). Money attitudes, credit card use, and compulsive buying among American college students. The Journal of Consumer Affairs, 35, 213-240.

Quintanilla, I., \& Bonavía, T. (2005). Psicología y economía. Valencia: Universitat de Valéncia.

Skinner, B. F. (1977). Ciencia y conducta bumana. Barcelona: Editorial Fontanella.

Van, G. M. (1979). European research in economic psychology. The Journal of Consumer Affairs, 13, 401-403.

Van Raaij, F. (1990). Economic psychology: Intersections in theory and application. Amsterdam: Elsevier Science Publishers.

Varela, J., Rial, A., Briana, T., García, C., \& Olea, J. (1996), Una medida de la implicación con las marcas comerciales. Psicothema, 8, 543-551.

Vidal, R, I. (1996). Is today right to buy? Analysis of the factors which shape this opinion. European Journal of Marketing, 30, 70-85.

Velandia, A. (2002). Modificación del posicionamiento de una marca, explicada a través de marcos relacionales. Acta Colombiana de Psicología, 8, 89-106.

Worchel, S., Cooper, J., Goethals, G., \& Olson, J. (2002). Psicología social. México: Thompson. 